Moller (Ed.)

Progress in Brain Research, Vol. 157

ISSN 0079-6123

Copyright (C) 2006 Elsevier B.V. All rights reserved

\title{
Cortical plasticity and rehabilitation
}

\author{
Raluca Moucha and Michael P. Kilgard*
}

Abstract: The brain is constantly adapting to environmental and endogenous changes (including injury) that occur at every stage of life. The mechanisms that regulate neural plasticity have been refined over millions of years. Motivation and sensory experience directly shape the rewiring that makes learning and neurological recovery possible. Guiding neural reorganization in a manner that facilitates recovery of function is a primary goal of neurological rehabilitation. As the rules that govern neural plasticity become better understood, it will be possible to manipulate the sensory and motor experience of patients to induce specific forms of plasticity. This review summarizes our current knowledge regarding factors that regulate cortical plasticity, illustrates specific forms of reorganization induced by control of each factor, and suggests how to exploit these factors for clinical benefit.

Keywords: Cortical plasticity; Experience-dependent plasticity; Cortical reorganization; Neuromodulators; Cholinergic; Rehabilitation

\section{Factors that regulate plasticity}

Plasticity is the remarkable ability of developing, adult, and aging brains to adapt to a changing world. This potential is revealed whenever an organism must meet a new environmental demand or recover from nervous system damage. Plasticity occurs in sensory and motor systems following deprivation of input or overstimulation, increased or decreased usage, learning of new skills, and injury. These experience-dependent changes can be as subtle as a change in neuronal excitability (Engineer et al., 2004) or as dramatic as the rewiring of auditory cortex to process visual information (Sur et al., 1988). Topographic maps, receptive field (RF) size, neuronal firing rate, temporal precision, and combination sensitivity can all be modified by our experiences. The types of plasticity activated by specific situations depend on the nature of the experiences and their behavioral sig-

\footnotetext{
*Corresponding author. Tel.: (972) 883-2345; Fax: (972) 8832491; E-mail: kilgard@utdallas.edu
}

nificance, conveyed by release of modulatory neurotransmitters (Fig. 1).

\section{Attentional modulation}

Neural plasticity is essential for adapting to changes in the environment but plasticity can be destabilizing if not well regulated. Limiting plasticity prevents meaningless events from driving changes that could degrade previously acquired memories and skills. Attention plays a key role in the regulation of plasticity associated with sensory experience. Repeated sensory stimulation alters topography in primary sensory cortex only when monkeys use the stimuli to make behavioral judgments (Recanzone et al., 1992, 1993). Many studies have shown that cortical neurons respond differently to attended versus unattended stimuli. Neurons in secondary somatosensory cortex, for example, exhibit greater response synchronization when monkeys are engaged in a tactile task (Steinmetz et al., 2000). Attention can also directly affect 
firing rates of cortical neurons (Treue and Maunsell, 1999; Recanzone and Wurtz, 2000). Results from several psychophysical studies support the hypothesis that attention regulates cortical plasticity and learning. Distinct forms of perceptual learning result when subjects attend to different features of an otherwise identical sensory input (Ahissar and Hochstein, 1993). Exposure to moving dot patterns can improve motion direction discrimination ability even if the motion is undetectable (due to low coherence), as long as the subjects are actively engaged in a visual task (Watanabe et al., 2001; Seitz and Watanabe, 2003). These results suggest that directed attention facilitates the learning of associated sensory features.

\section{Neuromodulatory influences}

Several neuromodulators, such as dopamine, norepinephrine, and acetylcholine, are known to regulate learning and memory in humans (Hasselmo, 1995). The observation that synaptic plasticity is also enhanced by the presence of these neurotransmitters supports the relationship between learning and plasticity (Singer, 1986; Brocher et al., 1992). Injection of acetylcholine or norepinephrine directly into visual, somatosensory, or auditory cortex during sensory stimulation can promote expression of neural plasticity in the intact brain (Greuel et al., 1988; McKenna et al., 1989; Delacour et al., 1990). Pairing sensory inputs with electrical activation of the nucleus basalis (NB), locus coeruleus (LC), or ventral tegmental area (VTA) also results in plasticity that is specific to features of the associated input (Kilgard and 
1 Merzenich, 1998; Bouret and Sara, 2002; Bao et al., 2003). Stimulation of neuromodulatory neurotransmitter release by amphetamine enhances cortical plasticity in human subjects (Dinse et al., 2003; Tegenthoff et al., 2004). Since release of these neurotransmitters is normally triggered by behaviorally arousing events, it is likely they contribute to the regulation of cortical plasticity.

\section{Patterns of sensory activation}

Many studies have shown that sensory input determines the form of cortical reorganization. When animals or humans repeatedly practice a skill that engages a limited region of the sensory epithelium, the regions of the cortical map that respond to task-specific inputs are enlarged (Jenkins et al., 1990; Recanzone et al., 1992, 1993; Elbert et al., 1995; Sterr et al., 1998). Cortical RFs can narrow or broaden and response latency can increase or decrease depending on the spatial and temporal pattern of sensory activation encountered during training. Owl monkeys trained on a tone frequency discrimination task have A1 neurons with smaller RFs and longer response latencies than untrained controls (Recanzone et al., 1993). Monkeys trained to detect changes in the rate of a tactile vibration exhibit larger RFs and faster response latencies (Recanzone et al., 1992). In contrast, training on a task with stimuli that move across the skin cause RFs to shrink (Jenkins et al., 1990). Training on a visual orientation task increased the steepness of orientation tuning in the trained region of the visual field (Schoups et al., 2001). These studies support the hypothesis that perceptual learning and cortical plasticity are specific to attended sensory features.

The rodent whisker system has proven particularly useful for directly comparing how cortical plasticity is shaped by different spatial patterns of activity. If all but one whisker is cut, for example, the responsiveness of the spared whisker is increased (Glazewski et al., 1998). Cutting a single whisker reduces input to the corresponding region of barrel cortex, decreases the responsiveness of the deprived neurons, and increases the responsiveness to neighboring whiskers. If all the whisk- ers are cut, the reduction in the response to the principle whisker is more modest. A checkerboard deprivation pattern causes responses to the deprived whiskers to decrease, but does not increase the response to the spared whiskers (Wallace and Fox, 1999). Finally, cutting all but two neighboring whiskers causes the RF of neurons in each region to shift toward the other spared whisker (Diamond et al., 1993). These results suggest that competition between sensory inputs induces the different forms of changes in responsiveness.

\section{Timing of sensory inputs}

The temporal coincidence of sensory stimulation can be just as important as its spatial pattern in determining the direction and magnitude of cortical plasticity. Inputs that are correlated in time are more likely to cause a change in neural responses than uncorrelated inputs. Simultaneous activation of an area of skin with a vibrating disc increased RF size in primary somatosensory cortex, while stimulation of a single point on the skin does not cause any change (Godde et al., 1996). Simultaneous activation of the developing auditory system by repeated exposure to broadband noise causes increased cortical RFs and degraded tonotopic maps (Chang and Merzenich, 2003). Such changes are not seen after equivalent exposure to tones. Increased simultaneous activation of the fingers due to surgical fusion or operant training leads to large, multidigit RFs in somatosensory cortex (Allard et al., 1991; Wang et al., 1995). This finding suggests that the usual segregation of each digit's cortical representation reflects the normally asynchronous activation of each digit. In vitro and more recently in vivo studies have further demonstrated that the time window for correlated inputs to induce plasticity is on the order of tens of milliseconds (Tsodyks, 2002; Dan and Poo, 2004). These results indicate that the precise spatial and temporal pattern of inputs shape cortical networks due to operation of Hebbian synaptic plasticity. 


\section{Duration of experience}

Many factors regulate the time course of learning and plasticity (Ebbinghaus, 1885; Dubnau et al., 2003). Fear conditioning can induce rapid and long lasting shifts of neuronal tuning toward the frequency of the conditioned tone (Bjordahl et al., 1998; Weinberger, 2003). In contrast, plasticity following skill learning or use-dependent plasticity develops gradually over time. The magnitude of effects often depends on duration of training and correlates with performance accuracy (Pleger et al., 2003). Motor map reorganization, which is accompanied by synaptogenesis and believed to underlie consolidation of motor skills, occurs during the late phase (after 10 days) of motor skill learning (Kleim et al., 2004). The schedule of inputs can also determine the induction of stable versus reversible synaptic modifications (Mauelshagen et al., 1998). Spaced repetition of LTP inducing stimuli prevents the reversal of LTP due to subsequent spontaneous activity that occurs after massed repetition (Zhou et al., 2003). Stable synaptic modifications are also induced by visual experience when the exposure to unidirectional moving bars occurs in a spaced pattern (three sets of 60 flashes separated by $5 \mathrm{~min}$ ) versus massed pattern (180 flashes continuously). If persistent synaptic changes are important for learning and memory, the effective use of training strategies that prevent their reversal is important. In behaviorally trained mice temporally spaced training more effectively recruits protein synthesis and enhanced long-term memory of contextual conditioning, while massed training triggers greater protein phosphatase 1 activity which suppresses memory formation (Genoux et al., 2002; Scharf et al., 2002). These results suggest that the schedule of training determines the duration of neural plasticity and learning.

\section{Influence of background stimuli on plasticity}

Psychologists and psychophysicists have known for decades that unattended background stimuli (context) influence perceptual learning. Studies of sensory plasticity have typically been conducted in environments stripped of context, by using soundproof booths or gray backgrounds. Recent experiments in more naturalistic and complex settings have shown that context also influences plasticity.

In many cases, adding complex backgrounds actually improves learning. Contrast discrimination learning, for example, can be facilitated by fixed contrast stimuli flanking the target stimulus (Adini et al., 2002). Dim line objects are easier to detect when flanked with a second collinear bar (Kapadia et al., 1995). This improvement in human performance is paralleled an enhancement of neuronal responses in monkey V1 when equivalent visual stimuli are presented (Kapadia et al., 1995). When foot shock is paired with a tone, the presence of unpaired background tones determines whether auditory cortex neurons shift their frequency tuning toward or away from the paired tone (Bakin and Weinberger, 1990; Ohl and Scheich, 1996; Dimyan and Weinberger, 1999).

While all of these studies suggest that many factors regulate plasticity and learning, direct comparison of the interactions between these factors has proven difficult. Differences in the behavioral response, task difficulty, task goal, motivation, modality and species often confound the influence of the discussed factors on plasticity. Because these factors are so tightly interdependent it has been difficult to tease apart their relative importance in directing different forms of plasticity. Varying sensory patterns or adding a complex background, for example, would also affect task difficulty in most cases. Currently, reduced preparations provide the best opportunities to study the interactions between each of the factors that regulate neural plasticity. Experimental paradigms that directly stimulate modulatory systems have proven particularly valuable in documenting the influence of stimulus pattern, timing, and background conditions on cortical plasticity.

\section{Sensory input paired with controlled release of neuromodulators}

Pairing electrical activation of the cholinergic NB with different sounds generates changes in cortical map and RF properties in rats that closely parallel 
1 the different forms of plasticity resulting from operant training in monkeys. For example, tem- porally modulated stimuli tend to increase RF size, while stimuli that activate different regions of the receptor surface tend to decrease RF size (Kilgard et al., 2002). While the differential plasticity observed in operant studies could be attributed to any number of technical differences, in the NB stimulation experiments the only explanation for the differential plasticity was the temporal and spectral properties of the sounds associated with NB stimulation. The observation that similar sensory inputs lead to comparable plasticity even in the absence of operant training supports the conclusion that sensory features determine the form of cortical plasticity.

During natural learning, changing task contingencies are known to alter the type, amount, and timing of neuromodulator release. For example, novel sounds activate cholinergic NB neurons for a few trials, but habituate rapidly (Richardson and DeLong, 1990, 1991). The response can later be reinstated if the sound is associated with a reward or punishment. NB releases acetylcholine onto the cortex only during the learning phase of a lever press task, but not after the task is well learned (Orsetti et al., 1996). Electrical stimulation bypasses the natural triggers of NB activity and eliminates the natural brake on cortical plasticity. The consistency of electrical activation makes it possible to systematically compare how the type, amount, and timing of neuromodulator release influence cortical plasticity when associated with sensory stimuli of differing spatial and temporal properties.
Single tone

Tone train

Distributed tones

Distributed tone trains

Frequency modulated tones

Complex acoustic sequence

Background sounds

\section{Patterns of activation determine type of reorganization}

Distinct types of cortical reorganization are generated when NB stimulation is associated with different sensory inputs. Cortical topography, RF size, and response timing are altered as a function of the temporal modulation and spatial distribution of inputs associated with NB stimulation. The focal activation caused by presentations of a single tone frequency results in expansion of the area responsive to the tone, and modest RFs broadening. Distributing the activation over more frequency sectors (i.e., seven tone frequencies) prevents the map reorganization but results in a narrowing of RFs (Kilgard et al., 2001). Rapidly modulated tone trains cause map expansion and dramatic RF broadening when activation is focal (i.e., one carrier frequency) and less extreme RF broadening and no map plasticity when the tone trains activate several regions (i.e., seven different carrier frequencies). These results document how different activation patterns direct cortical plasticity: (1) sensory map expansion only results when sensory activation is focal. (2) Distributing inputs across the cochlea tends to reduce RF size. (3) Modulated stimuli tend to increase RF size compared to unmodulated stimuli (Table 1, Kilgard et al., 2002). The observation that RF size is increased by stimuli with high degree of temporal modulation and little spatial variability (tone trains) and decreased by stimuli with high spatial variability and no temporal modulation (unmodulated tones of varying frequency) is consistent with earlier observations of plasticity in operant trained monkeys (Recanzone et al., 1992, 1993). These results indicate that NB stimulation directs changes that are

1

3

5

7

9

11

13

15

Map expansion + decrease latency

Map expansion + decrease latency $+\mathrm{RF}$ broadening

$\mathrm{RF}$ narrowing + increase latency

$\mathrm{RF}$ broadening + temporal plasticity

RF broadening + decrease latency + decreased thresholds

Combination sensitivity + decrease latency + decreased thresholds

Alters plasticity generated in silence
References

Kilgard and Merzenich (1998) Kilgard and Merzenich, 2001 QA:5 Kilgard and Merzenich (1998) Kilgard and Merzenich (2001) Moucha et al. (2005)

Kilgard and Merzenich (2002) Moucha et al. (2005) 
similar to operant induced plasticity even though the rats did not use the stimuli in any way.

Natural sounds usually vary both in spatial and temporal structure and create more complex activity patterns than tones. Pairing frequency modulated sweeps and complex acoustic sequences leads to forms of plasticity that are unpredictable from earlier studies with simple tones (Kilgard and

Merzenich, 2002; Moucha et al., 2005). FM sweeps result in a moving pattern of activation across the cochlea. Although tones and FM sweeps may share many features in common (including starting frequency, bandwidth, intensity, duration), pairing FM sweeps with NB stimulation causes different plasticity compared with unmodulated tones. Pairing FM sweeps with NB decreases response latency, broadens RFs, and increased sensitivity to quiet tones. These changes are restricted to the region of $\mathrm{A} 1$ activated by the sweep, but no map expansion results. When the starting frequency of the FM sweeps is varied no plasticity is observed in any region of A1 (Moucha et al., 2005). While repeated exposure to FM's does not cause any preference for FM direction (increasing or decreasing), pairing a sequence of sounds with identical NB stimulation can result in the development of responses sensitive to tone order.

Although plasticity mechanisms have presumably evolved to increase cortical processing capacity for behaviorally relevant inputs, it is not immediately obvious why the plasticity associated with each spatial and temporal input pattern is beneficial.

\section{Correlation of sensory inputs}

Studies in auditory, visual, and somatosensory cortex have suggested that input correlations strongly influence neural plasticity (Buonomano and Merzenich, 1998). In the developing visual system, for example, alternating asynchronous electrical stimulation of the optic nerve prevents normal development of binocular visual responses (Stryker and Strickland, 1984). In auditory cortex, sounds designed to decrease or increase correlation across the frequency map lead to very different forms of plasticity (Pandya et al., 2005). Alternat- ing activation of two nonoverlapping auditory neuron populations by two tones of distant frequencies (2 and $14 \mathrm{kHz}$ ) results in map segregation, decreased excitability, and longer response latencies of the activated neurons. These changes do not occur when NB-stimulation is paired with a modulated noise burst that synchronously activate large populations of A1 neurons. Pairing pulsed noises with NB stimulation disrupts tonotopic maps and reduces spontaneous discharge correlation in the primary auditory cortex (Bao et al., 2003). These finding are in agreement with the Hebbian postulate that inputs with decreased correlation weaken cortical responses and supports other observations that primary sensory cortices segregate inputs that are asynchronous and integrate correlated inputs (Allard et al., 1991; Wang et al., 1995).

\section{Duration of associative sensory pairing}

The duration of NB-induced plasticity depends on the schedule of the pairing protocol. Repetitively pairing NB stimulation with a tone for several minutes causes a shift in frequency tuning that reverses within $5 \mathrm{~h}$ (Zhang et al., 2005). Cortical map expansion builds with repeated pairings. One month of $300 \mathrm{NB}$-tone pairings per day increases the A1 representation of the paired frequency by twice as much as a week of pairing (Kilgard and Merzenich, 1998). After a month of pairing, NBinduced map plasticity endures for at least 20 days (Carrasco et al., 2004). NB stimulation also increases the duration of cortical and subcortical plasticity induced by cortical microstimulation (Ma and Suga, 2003). These results support earlier observations that cholinergic modulation contributes to both short-term and long-term plasticity.

\section{Background stimuli influence plasticity outcomes}

Although background stimuli are known to influence task performance and plasticity (Kapadia et al., 1995; Adini et al., 2002), it has not been clear whether the differences are due to altered task difficulty or to some specific influence of the dis- 
tracters. By directly pairing sensory stimuli with NB stimulation in different contexts, we have shown that background stimuli can influence plasticity independent of any influence on task performance.

Background sounds can alter bandwidth, threshold, and map plasticity. The $20 \%$ increase in RF size that occurs after pairing a single tone with NB stimulation does not occur if the same tone-NB pairing is interleaved with flanking tones that are not associated with NB stimulation (Kilgard et al., 2001). Repeated presentation of the word $/ \mathrm{SASH} /$ paired with $\mathrm{NB}$ stimulation causes expansion of the high frequency region of A1 (Pandya et al., 2003), presumably because the first element of the word is the high frequency $/ \mathrm{S} /$ sound. This map plasticity is eliminated when each phoneme of the word $/ \mathrm{S} /, / \mathrm{A} /$, and $/ \mathrm{SH} /$ are also presented, but not paired with NB stimulation. Finally, the addition of unpaired FM sweeps that contrast the duration and direction of the paired FM sweeps results in threshold and latency plasticity not observed if the identical FM's sweeps are paired with NB stimulation in a silent background (Moucha et al., 2005). These results indicate that background conditions, previously thought to be irrelevant, are likely to shape many forms of cortical plasticity.

\section{Clinical conclusions}

It was proposed two decades ago that cortical reorganization after injury may be the neural substrate for recovery of function after brain damage (Jenkins and Merzenich, 1987). More recent studies in primates have shown that rehabilitative training can direct reorganization to benefit recovery (Nudo et al., 1996). There is no longer a doubt that reorganization after brain lesions is shaped by the sensorimotor experiences in the weeks to months following injury. Hence it is important to effectively manage plasticity after brain damage. Many of the factors that influence plasticity can be manipulated in clinical settings to enhance therapeutic outcomes.

Attention is often impaired after brain injury and likely plays a critical role in directing training- induced plasticity. Patients with the highest vigilance scores typically receive greatest benefit from the rehabilitation therapy (Sohlberg et al., 2000). Some strategies, such as constraint therapy, that increase arousal (and even frustration) can be more effective than traditional occupational therapies (Taub and Uswatte, 2003).

The diffuse modulatory systems including the cholinergic NB are particularly vulnerable to dysregulation after brain damage. Experimental damage to the NB prevents map reorganization and retards skill learning in rats (Fig. 2). The recent observation that NB damage also prevents recovery from brain damage suggests many of the same mechanisms that regulate normal learning also regulate recovery from injury (Conner et al., 2005). In some patients, medication may be beneficial for normalizing attentional and neuromodulatory mechanisms. Agents that stimulate neuromodulators known to place the brain in a permissive state for experience-dependent changes are most likely to be effective.

Drugs that act on noradrenergic, dopaminergic, serotonergic, and cholinergic systems have been shown in laboratory and clinical research to be pharmacological adjuvants in neurorehabilitation (Phillips et al., 2003). Amphetamines lead to a diffuse increase of several modulators and can have a positive influence even when administered only as a single dose at the beginning of therapy (Feeney et al., 1982). It is important to note that drug administration only aids recovery when paired with practice. Amphetamine administration during speech language therapy increases the rate of improvement of aphasic patients during the early recovery period after stroke (Walker-Batson et al., 2004). Amphetamine also facilitates speech training in adult cochlear implant users (Tobey et al., 2005) and second language acquisition in normal subjects (Breitenstein et al., 2004). More research is needed to evaluate how best to facilitate neurological recovery using nervous system stimulants and other psychoactive compounds.

Since sensory and motor experiences (associated with release of modulatory neurotransmitters) determine the form of plasticity generated, it is critical to develop targeted rehabilitation techniques designed to stimulate adaptive plasticity following 
a) Before Training:

b) After Training:

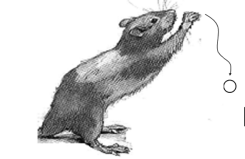

Poor reaching accuracy

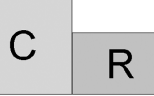

Small forepaw representation

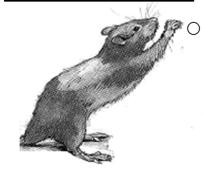

Good reaching accuracy

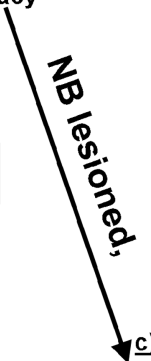

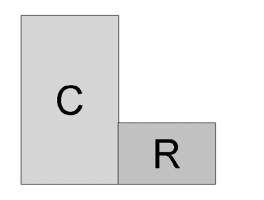

Large caudal forepaw representation
After Training:

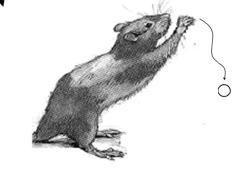

Poor reaching accuracy

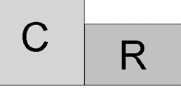

Small forepaw representation
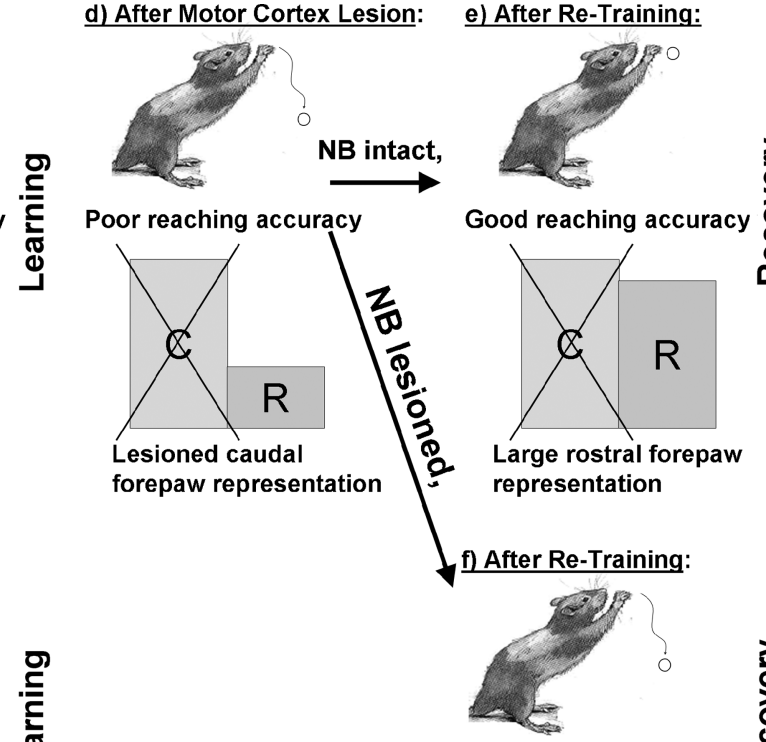

Poor reaching accuracy

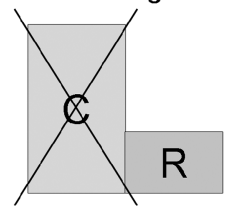

Small rostral forepaw representation

(Fraser et al., 2002). Several treatment strategies now effectively combine modulation of somatosensory input, administration of pharmacological adjuvants, and cortical stimulation to improve outcomes of rehabilitation (Hummel and Cohen, 2005).

The influence of background has not been wellstudied in the context of neurorehabilitation. However, studies have documented beneficial
1

3

5

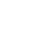

3

列

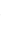


Table 2. Factors regulating plasticity

Factors regulating Effect

References

plasticity

Effect

\section{Pattern of stimuli \\ 7 \\ Temporal delivery (spaced vs. massed training)}

Attention

Drugs

Enhances stimulus driven plasticity via internal trigger of neuromodulator release

Achieve optimal levels of neuromodulators required for plasticity

Determines form of plasticity (reorganization of sensory representations, temporal precision, spatial selectivity, etc.)

Duration of training
Potentiation and stabilization of changes by stimulating protein synthesis mechanisms, and reducing phosphatases that prevent longterm changes

Consolidation of changes via synaptogenesis
Hasselmo (1995)

Phillips et al. (2003)

Buonomano and

Merzenich (1998)

Genoux et al. (2002);

Scharf et al. (2002); Zhou et al. (2003)

Kleim et al. (2004) transmitters, is essential to improving neurorehabilitation.

\section{Acknowledgments}

We thank Cherie Percaccio, Amanda Puckett, Vikram Jakkamsetti, Dr. Owen Floddy and Dr. Pritesh Pandya for insightful comments and review of the manuscript. This work was supported by grants from the National Institute for Deafness and Other Communication Disorders, the James S. McDonnell Foundation, and Cure Autism Now.

\section{References}

Adini, Y., Sagi, D. and Tsodyks, M. (2002) Context-enabled learning in the human visual system. Nature, 415: 790-793.

Ahissar, M. and Hochstein, S. (1993) Attentional control of early perceptual learning. Proc. Natl. Acad. Sci. USA,, 90: 5718-5722.

Allard, T., Clark, S.A., Jenkins, W.M. and Merzenich, M.M. (1991) Reorganization of somatosensory area 3b representations in adult owl monkeys after digital syndactyly. J. Neurophysiol., 66: 1048-1058.

Bakin, J.S. and Weinberger, N.M. (1990) Classical conditioning induces CS-specific receptive field plasticity in the auditory cortex of the guinea pig. Brain Res., 536: 271-286.

Bao, S., Chang, E.F., Davis, J.D., Gobeske, K.T. and Merzenich, M.M. (2003) Progressive degradation and subsequent refinement of acoustic representations in the adult auditory cortex. J. Neurosci., 23: 10765-10775.

Biernaskie, J. and Corbett, D. (2001) Enriched rehabilitative training promotes improved forelimb motor function and enhanced dendritic growth after focal ischemic injury. J. Neurosci., 21: 5272-5280. plasticity. Manipulation of the many parameters known to shape brain plasticity, including the pattern, timing, and duration of events associated 
Bjordahl, T.S., Dimyan, M.A. and Weinberger, N.M. (1998) Induction of long-term receptive field plasticity in the auditory cortex of the waking guinea pig by stimulation of the nucleus basalis. Behav. Neurosci., 112: 467-479.

Bouret, S. and Sara, S.J. (2002) Locus coeruleus activation modulates firing rate and temporal organization of odourinduced single-cell responses in rat piriform cortex. Eur. J. Neurosci., 16: 2371-2382.

Breitenstein, C., Wailke, S., Bushuven, S., Kamping, S., Zwitserlood, P., Ringelstein, E.B. and Knecht, S. (2004) Damphetamine boosts language learning independent of its cardiovascular and motor arousing effects. Neuropsychopharmacology, 29: 1704-1714.

Brocher, S., Artola, A. and Singer, W. (1992) Agonists of cholinergic and noradrenergic receptors facilitate synergistically the induction of long-term potentiation in slices of rat visual cortex. Brain Res., 573: 27-36.

Buonomano, D.V. and Merzenich, M.M. (1998) Cortical plasticity: from synapses to maps. Annu. Rev. Neurosci., 21: 149-186.

Carrasco, R.A., Puckett, A., Pandya, P.K., McMenamy, A., Gibbons, J., Heydrick, C., Moucha, R., Jain, R. and Kilgard, M.P. (2004). Frequency map reorganization in rat primary auditory cortex enabled by basal forebrain activation endures for at least 20 days. In: Society for Neuroscience, San Diego, CA.

Chang, E.F. and Merzenich, M.M. (2003) Environmental noise retards auditory cortical development. Science, 300: 498-502.

Conner, J.M., Chiba, A.A. and Tuszynski, M.H. (2005) The basal forebrain cholinergic system is essential for cortical plasticity and functional recovery following brain injury. Neuron, 46: 173-179.

Dan, Y. and Poo, M.M. (2004) Spike timing-dependent plasticity of neural circuits. Neuron, 44: 23-30.

Delacour, J., Houcine, O. and Costa, J.C. (1990) Evidence for a cholinergic mechanism of "learned" changes in the responses of barrel field neurons of the awake and undrugged rat. Neuroscience, 34: 1-8.

Diamond, M.E., Armstrong-James, M. and Ebner, F.F. (1993) Experience-dependent plasticity in adult rat barrel cortex. Proc. Natl. Acad. Sci. USA, 90: 2082-2086.

Dimyan, M.A. and Weinberger, N.M. (1999) Basal forebrain stimulation induces discriminative receptive field plasticity in the auditory cortex. Behav. Neurosci., 113: 691-702.

Dinse, H.R., Ragert, P., Pleger, B., Schwenkreis, P. and Tegenthoff, M. (2003) Pharmacological modulation of perceptual learning and associated cortical reorganization. Science, 301 : 91-94.

Dubnau, J., Chiang, A.S. and Tully, T. (2003) Neural substrates of memory: from synapse to system. J. Neurobiol., 54: 238-253.

Ebbinghaus, H. (1885) Uber das Gedachtnis. Untersuchungen zur Experimentellen Psychologie. Duckner \& Humbolt, Leipzig.

Elbert, T., Pantev, C., Wienbruch, C., Rockstroh, B. and Taub, E. (1995) Increased cortical representation of the fingers of the left hand in string players. Science, 270: 305-307.
Engineer, N.D., Percaccio, C.R., Pandya, P.K., Moucha, R., Rathbun, D.L. and Kilgard, M.P. (2004) Environmental enrichment improves response strength, threshold, selectivity, and latency of auditory cortex neurons. J. Neurophysiol., 92: 73-82.

Feeney, D.M., Gonzalez, A. and Law, W.A. (1982) Amphetamine, haloperidol, and experience interact to affect rate of recovery after motor cortex injury. Science, 217: 855-857.

Fraser, C., Power, M., Hamdy, S., Rothwell, J., Hobday, D., Hollander, I., Tyrell, P., Hobson, A., Williams, S. and Thompson, D. (2002) Driving plasticity in human adult motor cortex is associated with improved motor function after brain injury. Neuron, 34: 831-840.

Genoux, D., Haditsch, U., Knobloch, M., Michalon, A., Storm, D. and Mansuy, I.M. (2002) Protein phosphatase 1 is a molecular constraint on learning and memory. Nature, 418: 970-975.

Glazewski, S., McKenna, M., Jacquin, M. and Fox, K. (1998) Experience-dependent depression of vibrissae responses in adolescent rat barrel cortex. Eur. J. Neurosci., 10: 2107-2116.

Godde, B., Spengler, F. and Dinse, H.R. (1996) Associative pairing of tactile stimulation induces somatosensory cortical reorganization in rats and humans. Neuroreport., 8: 281-285.

Greuel, J.M., Luhmann, H.J. and Singer, W. (1988) Pharmacological induction of use-dependent receptive field modifications in the visual cortex. Science, 242: 74-77.

Hasselmo, M.E. (1995) Neuromodulation and cortical function: modeling the physiological basis of behavior. Behav. Brain Res., 67: 1-27.

Hummel, F.C. and Cohen, L.G. (2005) Drivers of brain plasticity. Curr. Opin. Neurol., 18: 667-674.

Jenkins, W.M. and Merzenich, M.M. (1987) Reorganization of neocortical representations after brain injury: a neurophysiological model of the bases of recovery from stroke. Prog. Brain Res., 71: 249-266.

Jenkins, W.M., Merzenich, M.M., Ochs, M.T., Allard, T. and Guic-Robles, E. (1990) Functional reorganization of primary somatosensory cortex in adult owl monkeys after behaviorally controlled tactile stimulation. J. Neurophysiol., 63: 82-104.

Johansson, B.B. (2004) Brain plasticity in health and disease. Keio J. Med., 53: 231-246.

Kapadia, M.K., Ito, M., Gilbert, C.D. and Westheimer, G. (1995) Improvement in visual sensitivity by changes in local context: parallel studies in human observers and in V1 of alert monkeys. Neuron, 15: 843-856.

Kilgard, M.P. and Merzenich, M.M. (1998) Cortical map reorganization enabled by nucleus basalis activity. Science, 279: 1714-1718.

Kilgard, M.P. and Merzenich, M.M. (2002) Order sensitive plasticity in adult primary auditory cortex. Proc. Natl. Acad. Sci. USA, 99: 3205-3209.

Kilgard, M.P., Pandya, P.K., Engineer, N.D. and Moucha, R. (2002) Cortical network reorganization guided by sensory input features. Biol. Cybern., 87: 333-343.

Kilgard, M.P., Pandya, P.K., Vazquez, J., Gehi, A., Schreiner, C.E. and Merzenich, M.M. (2001) Sensory input directs spa- 
tial and temporal plasticity in primary auditory cortex. J. Neurophysiol., 86: 326-338.

Kleim, J.A., Hogg, T.M., VandenBerg, P.M., Cooper, N.R., Bruneau, R. and Remple, M. (2004) Cortical synaptogenesis and motor map reorganization occur during late, but not early, phase of motor skill learning. J. Neurosci., 24: 628-633.

Ma, X. and Suga, N. (2003) Augmentation of plasticity of the central auditory system by the basal forebrain and/or somatosensory cortex. J. Neurophysiol., 89: 90-103.

Mauelshagen, J., Sherff, C.M. and Carew, T.J. (1998) Differential induction of long-term synaptic facilitation by spaced and massed applications of serotonin at sensory neuron synapses of Aplysia californica. Learn. Mem., 5: 246-256.

McKenna, T.M., Ashe, J.H. and Weinberger, N.M. (1989) Cholinergic modulation of frequency receptive fields in auditory cortex: I. Frequency-specific effects of muscarinic agonists. Synapse, 4: 30-43.

Moucha, R., Pandya, P.K., Engineer, N.D., Rathbun, D.L. and Kilgard, M.P. (2005) Background sounds contribute to spectrotemporal plasticity in primary auditory cortex. Exp. Brain Res., 162: 417-427.

Nudo, R.J. (1997) Remodeling of cortical motor representations after stroke: implications for recovery from brain damage. Mol. Psychiatry,, 2: 188-191.

Nudo, R.J., Wise, B.M., SiFuentes, F. and Milliken, G.W. (1996) Neural substrates for the effects of rehabilitative training on motor recovery after ischemic infarct. Science, 272: 1791-1794.

Ohl, F.W. and Scheich, H. (1996) Differential frequency conditioning enhances spectral contrast sensitivity of units in auditory cortex (field $\mathrm{Al}$ ) of the alert Mongolian gerbil. Eur. J. Neurosci., 8: 1001-1017.

Orsetti, M., Casamenti, F. and Pepeu, G. (1996) Enhanced acetylcholine release in the hippocampus and cortex during acquisition of an operant behavior. Brain Res., 724: 89-96.

Pandya, P.K., Engineer, N., Moucha, R., Rathbun, D.L., Vazquez, J. and Kilgard, M.P. (2003). Background stimuli control cortical map expansion in primary auditory cortex. In: International Conference on Auditory Cortex, Magdeburg, Germany.

Pandya, P.K., Moucha, R., Engineer, N.D., Rathbun, D.L., Vazquez, J. and Kilgard, M.P. (2005) Asynchronous inputs alter excitability, spike timing, and topography in primary auditory cortex. Hear. Res., 203: 10-20.

Phillips, J.P., Devier, D.J. and Feeney, D.M. (2003) Rehabilitation pharmacology: bridging laboratory work to clinical application. J. Head Trauma Rehabil., 18: 342-356.

Pleger, B., Foerster, A.F., Ragert, P., Dinse, H.R., Schwenkreis, P., Malin, J.P., Nicolas, V. and Tegenthoff, M. (2003) Functional imaging of perceptual learning in human primary and secondary somatosensory cortex. Neuron, 40: 643-653.

Puurunen, K., Jolkkonen, J., Sirvio, J., Haapalinna, A. and Sivenius, J. (2001) Selegiline combined with enriched-environment housing attenuates spatial learning deficits following focal cerebral ischemia in rats. Exp. Neurol., 167: 348-355.

Recanzone, G.H., Merzenich, M.M., Jenkins, W.M., Grajski, K.A. and Dinse, H.R. (1992) Topographic reorganization of the hand representation in cortical area $3 \mathrm{~b}$ owl monkeys trained in a frequency-discrimination task. J. Neurophysiol., 67: 1031-1056.

Recanzone, G.H., Schreiner, C.E. and Merzenich, M.M. (1993) Plasticity in the frequency representation of primary auditory cortex following discrimination training in adult owl monkeys. J. Neurosci., 13: 87-103.

Recanzone, G.H. and Wurtz, R.H. (2000) Effects of attention on MT and MST neuronal activity during pursuit initiation. J. Neurophysiol., 83: 777-790.

Richardson, R.T. and DeLong, M.R. (1990) Context-dependent responses of primate nucleus basalis neurons in a go/nogo task. J. Neurosci., 10: 2528-2540.

Richardson, R.T. and DeLong, M.R. (1991) Electrophysiological studies of the functions of the nucleus basalis in primates. Adv. Exp. Med. Biol., 295: 233-252.

Scharf, M.T., Woo, N.H., Lattal, K.M., Young, J.Z., Nguyen, P.V. and Abel, T. (2002) Protein synthesis is required for the enhancement of long-term potentiation and long-term memory by spaced training. J. Neurophysiol., 87: 2770-2777.

Schoups, A., Vogels, R., Qian, N. and Orban, G. (2001) Practising orientation identification improves orientation coding in V1 neurons. Nature, 412: 549-553.

Seitz, A.R. and Watanabe, T. (2003) Psychophysics: is subliminal learning really passive? Nature, 422: 36.

Singer, W. (1986) The brain as a self-organizing system. Eur. Arch. Psychiatry Neurol. Sci., 236: 4-9.

Sohlberg, M.M., McLaughlin, K.A., Pavese, A., Heidrich, A. and Posner, M.I. (2000) Evaluation of attention process training and brain injury education in persons with acquired brain injury. J. Clin. Exp. Neuropsychol., 22: 656-676.

Steinmetz, P.N., Roy, A., Fitzgerald, P.J., Hsiao, S.S., Johnson, K.O. and Niebur, E. (2000) Attention modulates synchronized neuronal firing in primate somatosensory cortex. Nature, 404: 187-190.

Sterr, A., Muller, M.M., Elbert, T., Rockstroh, B., Pantev, C. and Taub, E. (1998) Changed perceptions in Braille readers. Nature, 391: 134-135.

Stryker, M. and Strickland, S. (1984) Physiological segregation of ocular dominance columns depends on the pattern of afferent electrical activity. Invest. Ophtamol. Vis. Sci. Suppl., 25: 278.

Sur, M., Garraghty, P.E. and Roe, A.W. (1988) Experimentally induced visual projections into auditory thalamus and cortex. Science, 242: 1437-1441.

Taub, E. and Uswatte, G. (2003) Constraint-induced movement therapy: bridging from the primate laboratory to the stroke rehabilitation laboratory. J. Rehabil. Med., 41: 34-40.

Tegenthoff, M., Cornelius, B., Pleger, B., Malin, J.P. and Schwenkreis, P. (2004) Amphetamine enhances training-induced motor cortex plasticity. Acta. Neurol. Scand., 109: 330-336.

Tobey, E.A., Devous Sr, M.D., Buckley, K., Overson, G., Harris, T., Ringe, W. and Martinez-Verhoff, J. (2005) Pharmacological enhancement of aural habilitation in adult cochlear implant users. Ear Hear., 26: 45S-56S. 
Treue, S. and Maunsell, J.H. (1999) Effects of attention on the processing of motion in macaque middle temporal and medial superior temporal visual cortical areas. J. Neurosci., 19: 7591-7602.

Tsodyks, M. (2002) Spike-timing-dependent synaptic plasticity - the long road towards understanding neuronal mechanisms of learning and memory. Trends Neurosci., 25: 599-600.

Walker-Batson, D., Smith, P., Curtis, S. and Unwin, D.H. (2004) Neuromodulation paired with learning dependent practice to enhance post stroke recovery? Restor. Neurol. Neurosci., 22: 387-392.

Wallace, H. and Fox, K. (1999) The effect of vibrissa deprivation pattern on the form of plasticity induced in rat barrel cortex. Somatosens. Mot. Res., 16: 122-138.

Wang, X., Merzenich, M.M., Sameshima, K. and Jenkins, W.M. (1995) Remodelling of hand representation in adult cortex determined by timing of tactile stimulation. Nature, 378: $71-75$.
Watanabe, T., Nanez, J.E. and Sasaki, Y. (2001) Perceptual learning without perception. Nature, 413: 844-848.

Weinberger, N.M. (2003) The nucleus basalis and memory codes: auditory cortical plasticity and the induction of specific, associative behavioral memory. Neurobiol. Learn Mem., 80: 268-284.

Xerri, C., Merzenich, M.M., Peterson, B.E. and Jenkins, W. (1998) Plasticity of primary somatosensory cortex paralleling sensorimotor skill recovery from stroke in adult monkeys. J. Neurophysiol., 79: 2119-2148.

Zhang, Y., Hamilton, S.E., Nathanson, N.M. and Yan, J. (2005). Decreased input-specific plasticity of the auditory cortex in mice lacking M1 muscarinic acetylcholine receptors. Cereb. Cortex, In press.

Zhou, Q., Tao, H.W. and Poo, M.M. (2003) Reversal and stabilization of synaptic modifications in a developing visual system. Science, 300: 1953-1957.
1

3 


\section{AUTHOR QUERY FORM}

\section{ELSEVIER}

\section{Progress in Brain \\ Research, Volume 157}

JOURNAL TITLE: PBR-V157

ARTICLE NO: $\mathbf{5 7 0 0 7}$

\section{Queries and / or remarks}

\begin{tabular}{|l|l|l|}
\hline $\begin{array}{c}\text { Query } \\
\text { No }\end{array}$ & \multicolumn{1}{|c|}{ Details required } & Author's response \\
\hline AQ1 & $\begin{array}{l}\text { Please provide book title and name of the editors for ref. } \\
\text { Carrasco et al. (2004) in the reference list }\end{array}$ & \\
\hline AQ2 & $\begin{array}{l}\text { Please provide the vol. and page range in the ref. Zhang et al. } \\
\text { (2005). }\end{array}$ & \\
\hline AQ3 & $\begin{array}{l}\text { The reference McEwen (2003) has not been included in } \\
\text { the reference list. Please provide. }\end{array}$ & \\
\hline AQ4 & $\begin{array}{l}\text { In Figure 2, label (a) description is not given. Please } \\
\text { include the description for label (a) as for labels (b)-(f). }\end{array}$ & \\
\hline AQ5 & $\begin{array}{l}\text { The reference Kilgard and Merzenich (2001) has not } \\
\text { been included in the reference list. Please provide. }\end{array}$ & \\
\hline UC REF & $\begin{array}{l}\text { If references appear under section “Uncited References", then cite } \\
\text { at relevant places in the text. In case of nonavailability of citation, } \\
\text { the corresponding references will be deleted from the reference list. } \\
\text { Please ignore this query if there is no 'Uncited Reference' section. }\end{array}$ & \\
\hline & & \\
\hline & & \\
\hline & & \\
\hline & & \\
\hline
\end{tabular}




\section{AUTHOR QUERY FORM}

ELSEVIER

\section{Progress in Brain Research, Volume 157}

\begin{tabular}{|l|l|}
\hline JOURNAL TITLE: & PBR-V157 \\
\hline ARTICLE NO: & $\mathbf{5 7 0 0 7}$ \\
\hline
\end{tabular}

Queries and / or remarks

\begin{tabular}{|c|c|c|}
\hline $\begin{array}{c}\text { Query } \\
\text { No }\end{array}$ & Details required & Author's response \\
\hline & & \\
\hline
\end{tabular}

